\title{
Hiss and snort call types of wild-living giraffes Giraffa camelopardalis: acoustic structure and context
}

\author{
Elena V. Volodina', Ilya A. Volodin ${ }^{1,2^{*}} \mathbb{0}$, Elena V. Chelysheva ${ }^{3}$ and Roland Frey ${ }^{4}$
}

\begin{abstract}
Objectives: Vocalization as part of vigilance behaviour is widespread across animal taxa, including ruminants. Calls of wild-living giraffes have never been recorded and spectrographically investigated. This study reports the acoustic structure of vigilance-related hiss and snort calls of wild-living giraffes Giraffa camelopardalis.

Results: The hiss and snort calls were emitted during five recording sessions produced by nine individual giraffes ( 8 adults and 1 subadult) in their natural environment in Namibia (3 individuals) and Kenya (6 individuals). These calls attended vigilance behaviour toward humans in hides or in vehicles and cheetahs as natural predators of giraffe young. This study provides spectrographic analyses of 22 hiss and 20 snort calls. The giraffe hisses were broadband vocalizations of an average duration of $0.72 \mathrm{~s}$ (from 0.24 to $1.04 \mathrm{~s}$ ) and a peak frequency of $0.69 \mathrm{kHz}$. The giraffe snorts were broadband pulsed calls of an average duration of $0.28 \mathrm{~s}$ (from 0.13 to $0.55 \mathrm{~s}$ ), a peak frequency at $0.20 \mathrm{kHz}$ and comprised a prominent low-frequency pulsation of 23.7 pulses/s. The acoustic structure of giraffe hisses is reminiscent of vigilance-related hisses of musk deer Moschus moschiferus. Giraffe snorts differ from snorts of other ruminants by their prominent pulsed pattern.
\end{abstract}

Keywords: Mammal, Acoustic communication, Vocalization, Emotional arousal, Giraffe, Hiss, Snort, Ungulate, Vigilance behaviour, Ruminant, Giraffa camelopardalis

\section{Introduction}

Vocalizations attend vigilance behaviour in many animal taxa [1]. The acoustic structure of these vocalizations may encode behavioural context and type of predator [25 ] as well as the degree of negative emotional arousal of the caller $[6,7]$ and thus the threat urgency [8-10]. These acoustic cues to the degree of urgency and arousal can be used for avoiding danger by conspecifics $[8,11,12]$ or heterospecifics [13, 14].

In mammals, vigilance-related vocalizations are best studied for group-living rodents [15, 16], primates [5, 17] and carnivores [18-20], and have been reported for fourteen species of ruminants [21-42], including giraffe Giraffa camelopardalis [38-42]. Among reported audible

\footnotetext{
*Correspondence: volodinsvoc@gmail.com

2 Department of Vertebrate Zoology, Faculty of Biology, Lomonosov

Moscow State University, Moscow 119234, Russia

Full list of author information is available at the end of the article
}

[38-40] and infrasonic (14 Hz) vocalizations [41, 42], only presence of audible call types (snort, burst, grunt and humm) was confirmed by recent studies of captive giraffes $[43,44]$.

Giraffe hisses were verbally reported as nasal calls emitted in the context of approach investigation [38]. Giraffe snorts were verbally reported as short plosive grunts, being produced through the widened nostrils while the animal was standing and scanning one particular spot or direction as a reaction to a potentially threatening irritation [45-47] or immediately after a dominant giraffe bull had chased off an inferior bull [47]. Giraffe snorts were reported as an illustrative spectrogram and a wave-file based on recordings made in captivity using automate recording systems [43]; acoustic analyses of these calls were not provided. Calls of wild-living giraffes have never been recorded previously. The purpose of this study was to present the acoustic structure of the hiss and snort calls of wild-living giraffes. 


\section{Main text}

\section{Methods}

Giraffe calls were collected in two natural localities: in February 2016 at the 15,000-hectare Okambara Ranch located $130 \mathrm{~km}$ east of Windhoek (Namibia) and in March and November-December 2016 in Maasai-Mara National Reserve and the adjacent area Mara Conservancy area (Kenya). During five recording sessions (2 in Namibia and 3 in Kenya, all conducted on different days), calls of 9 giraffes were recorded (3 individuals G.c. giraffa in Namibia and 6 individuals G.c. tippelskirchi in Kenya [48]), including 2 adult males, 2 adult females, 4 adults of unspecified sex and 1 subadult male.

For acoustic recordings $(48 \mathrm{kHz}, 16 \mathrm{bit}$, frequency range 20-20,000 Hz) we used Marantz PMD-660 or PMD661 solid state recorders (D\&M Professional, Kanagawa, Japan) with Sennheiser K6-ME66 or ME67 microphones (Sennheiser electronic, Wedemark, Germany). During recording, each call was labeled by the researcher's voice to identify the target vocalizations against background noises, the behavioural context and (where possible), the individual identity, sex and age of a caller. All calls were produced spontaneously by the giraffes; researchers did not provoke the animals to vocalize.

At the Namibia locality calls were recorded from hides during daytime and at twilight. One hide was a potential feeding tree of giraffes with overhanging branches that covered the researcher thus preventing his visual detection by the giraffes. Another hide consisted of a row of dense bushes, approached by giraffes at twilight in the evening when moving to a nearby water place for drinking. The researcher sitting in this hide was partly visible to the giraffes at a distance of approximately $20 \mathrm{~m}$.

In the Kenya locality, where leaving vehicles is prohibited, calls were recorded from a standing car during daytime at distance of $10-100 \mathrm{~m}$ to the giraffes. Two recordings were done when cheetahs Acinonyx jubatus, occasional predators of giraffe young $[49,50]$, were at $30-100 \mathrm{~m}$ to the target giraffes. The third recording was done on giraffe vigilance behaviour toward a standing car, in the absence of predators.

Calls of good quality were selected for acoustic analyses $(24 \mathrm{kHz}$ sampling frequency, Hamming window, FFT 1024 points, frame 50\%, overlap 93.75\%) using Avisoft SASLab Pro software (Avisoft Bioacoustics, Berlin, Germany). Based on the acoustic structure (Fig. 1) and the sounding (Additional file 1: Audio S1), we classified the calls into hiss and snort call types. For each call, we measured call duration from the screen with the standard marker cursor in the spectrogram window. After high-pass filtration $(100 \mathrm{~Hz}$, Gauss filter) we measured the maximum amplitude frequency (fpeak) and three quartiles (q25, q50 and q75), covering 25, 50 and 75\% of call energy from the mean power spectrum of each call. For the snorts (all of them displayed low-frequency pulsation), we measured the pulse rate with the standard marker cursor. All measurements were exported to Microsoft Excel (Microsoft Corp., Redmond, WA, USA) for analyses. In total, we measured 22 hiss calls (21 from Namibia and one from Kenya) and 20 snort calls ( 1 from Namibia and 19 from Kenya), from 1 to 11 (mean $\pm \mathrm{SD}=4.7 \pm 3.9$ ) calls per individual.

Statistical analyses were made with STATISTICA, v. 8.0 (StatSoft, Tulsa, OK, USA); all means are given as mean $\pm \mathrm{SD}$, and differences were considered significant whenever $p<0.05$. Nine of ten distributions did not depart from normality (Kolmogorov-Smirnov test, $p>0.05$ ), so, we could use Student $t$ test with Bonferroni correction for multiple comparisons to compare the parameter values between the hiss and snort calls.

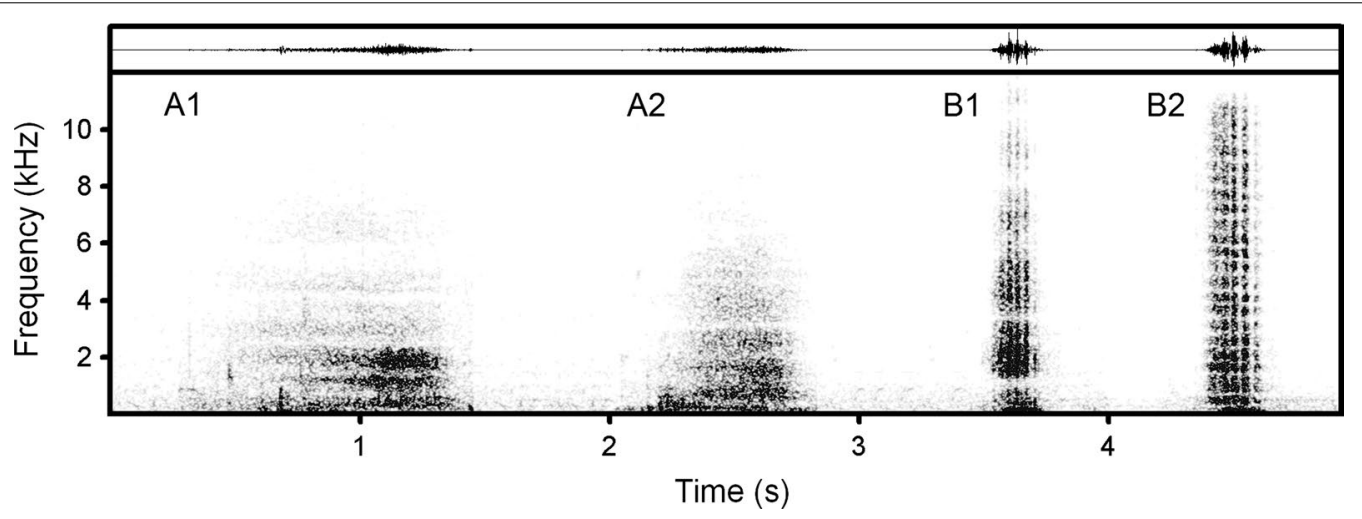

Fig. 1 Two vigilance-related call types in the giraffe Giraffa camelopardalis. The spectrogram (below) and waveform (above) illustrate (A1) hiss of an adult giraffe of unspecified sex in Namibia; $\mathbf{A} 2$ hiss of a subadult male giraffe in Kenya; B1 snort of an adult giraffe of unspecified sex in Namibia; B2 snort of an adult female in Kenya. In the waveform of the snorts, the low-frequency pulsation is visible as amplitude peaks. The spectrogram was created with $24 \mathrm{kHz}$ sampling frequency, Hamming window, FFT 1024 points, frame 50\% and overlap 93.75\%. Original wav-files are available in the electronic supporting information (Additional file 1: Audio S1) 


\section{Results}

Giraffe hisses were broadband vocalizations of duration $0.24-1.04 \mathrm{~s}$ and the peak frequency at $0.69 \pm 0.61 \mathrm{kHz}$ (Fig. 1, Table 1, Additional file 2: Table S1). Giraffe snorts were broadband vocalizations of duration 0.13-0.55 s and the peak frequency at $0.20 \pm 0.29 \mathrm{kHz}$, displaying a prominent low-frequency pulsation ranging in rate from 18.3 to 32.8 pulses/s between calls (Fig. 1, Table 1). Compared to hisses, snorts were shorter, their peak frequency was lower, and the lower, medium and upper power quartiles were all higher (Table 1).

We observed that snorts were produced nasally by a sudden burst of air released from the nostrils. However, we could not establish whether the hisses were produced through the nose or via the opened mouth. Emission of hisses toward a hidden researcher was sometimes preceded by neck-shaking. Emissions of snorts were not preceded or accompanied by any prominent movements; only one adult female nodded during the emission of snorts. Hisses and snorts were either produced singly or in series lasting more than half an hour. Hisses and snorts could occur in the same call series. Calls within series were separated by time intervals of up to a few minutes.

Most hisses (20 of 22) were produced toward a researcher, hidden under the overhanging tree branches. At the day of the recording, this hide was approached by a group of 7 giraffes for feeding. The giraffes came so close that their heads and parts of their bodies were 3-5 $\mathrm{m}$ above the researcher. Only then the animals detected the researcher and started the emission of hisses. The researcher could see the giraffe heads through the branches against the bright sky, whereas the giraffes, despite their acute vision [51], could hardly see the researcher when looking from bright sunlight towards the dense foliage. Probably, the hissing was triggered by the sudden detection of human smell just underneath their food source, as the olfactory abilities of giraffes are good [52]. Despite hampered vision of the researcher at least two callers could be identified because calls arrived from different sides at the researcher. After some time, five giraffes went off, whereas two animals stayed at a distance of $8 \mathrm{~m}$ and one of them continued hissing. The caller passed a few steps from one side to the other in front of the hiding tree and then hissed. Before hissing, the caller often displayed neck-shaking.

Most giraffe snorts (19 of 20) were recorded, when the callers were in $30-100 \mathrm{~m}$ of female cheetahs with cubs. Cheetahs were active, either eating a kill or moving along groups of giraffes but did not pay evident attention to the giraffes. During one recording, an adult giraffe from a group of three animals spotted a group of three cheetahs (a mother with two cubs) eating a kill and started approaching them slowly. The giraffe produced three snorts, the first snort at a distance of 50-60 m, the second at $45-50 \mathrm{~m}$ and the third at more than $100 \mathrm{~m}$, while retreating from the cheetahs. Intervals between the snorts were about $1 \mathrm{~min}$. During another recording, a single subadult male produced a long series of snorts and one hiss after an adult cheetah female had passed by and the giraffe ran off for a few meters and then stopped, looking at her.

\section{Discussion}

This study is the first describing the acoustic variables of calls of wild-living giraffes. Both the hiss and snort call types were rather soft and probably communicated vigilance at short range, as short-range ultrasonic alarm calls of ground squirrels [53, 54]. Whereas most hisses were recorded in Namibia and most snorts in Kenya, this discrepancy was probably related to the situation of higher potential danger and less predictability for giraffe callers in Namibia (where giraffes are legally hunted from the hides) than in Kenya (where the hunting is prohibited) and not to locality per se. Otherwise, the single hiss that was recorded in Kenya, was produced by a subadult male toward the cheetahs, that predate young giraffes $[49,50]$. Thus, hisses prevailed in the contexts of higher tenseness/unexpectedness for a caller whereas the snorts prevailed in the contexts of less unexpectedness for a caller. Two different call types in response to different levels of threat are known for many species of mammals and birds [55].

We took one single captive giraffe snort from the supplementary material of the study of Baotic et al. [43]

Table 1 Values (mean \pm SD) of acoustic variables measured for hiss and snort call types of giraffe and Student $\boldsymbol{t}$ test results of their comparison

\begin{tabular}{|c|c|c|c|c|c|c|}
\hline Call types & Duration (s) & fpeak (kHz) & q25 (kHz) & q50 (kHz) & q75 (kHz) & Pulse rate $(\mathrm{Hz})$ \\
\hline Hiss $(n=22)$ & $0.72 \pm 0.22$ & $0.69 \pm 0.61$ & $0.71 \pm 0.24$ & $1.55 \pm 0.37$ & $2.94 \pm 0.60$ & Non-pulsed \\
\hline Snort $(n=20)$ & $0.28 \pm 0.10$ & $0.20 \pm 0.29$ & $1.05 \pm 0.47$ & $2.23 \pm 0.90$ & $4.39 \pm 1.64$ & $23.7 \pm 4.2$ \\
\hline Student $t$ test results & $\begin{array}{l}t=8.34 \\
\boldsymbol{p}<\mathbf{0 . 0 0 1}\end{array}$ & $\begin{array}{l}t=3.11 \\
\boldsymbol{p}=\mathbf{0 . 0 0 3}\end{array}$ & $\begin{array}{l}t=3.03 \\
\boldsymbol{p}=\mathbf{0 . 0 0 4}\end{array}$ & $\begin{array}{l}t=3.21 \\
\boldsymbol{p}=\mathbf{0 . 0 0 3}\end{array}$ & $\begin{array}{l}t=3.84 \\
\boldsymbol{p}<\mathbf{0 . 0 0 1}\end{array}$ & Not applicable \\
\hline
\end{tabular}

Designations: duration—call duration; fpeak_peak frequency; q25, q50 q75-lower, medium and upper quartiles. $p$ estimates less than 0.01 (after Bonferroni correction) are shown in bolditalics 

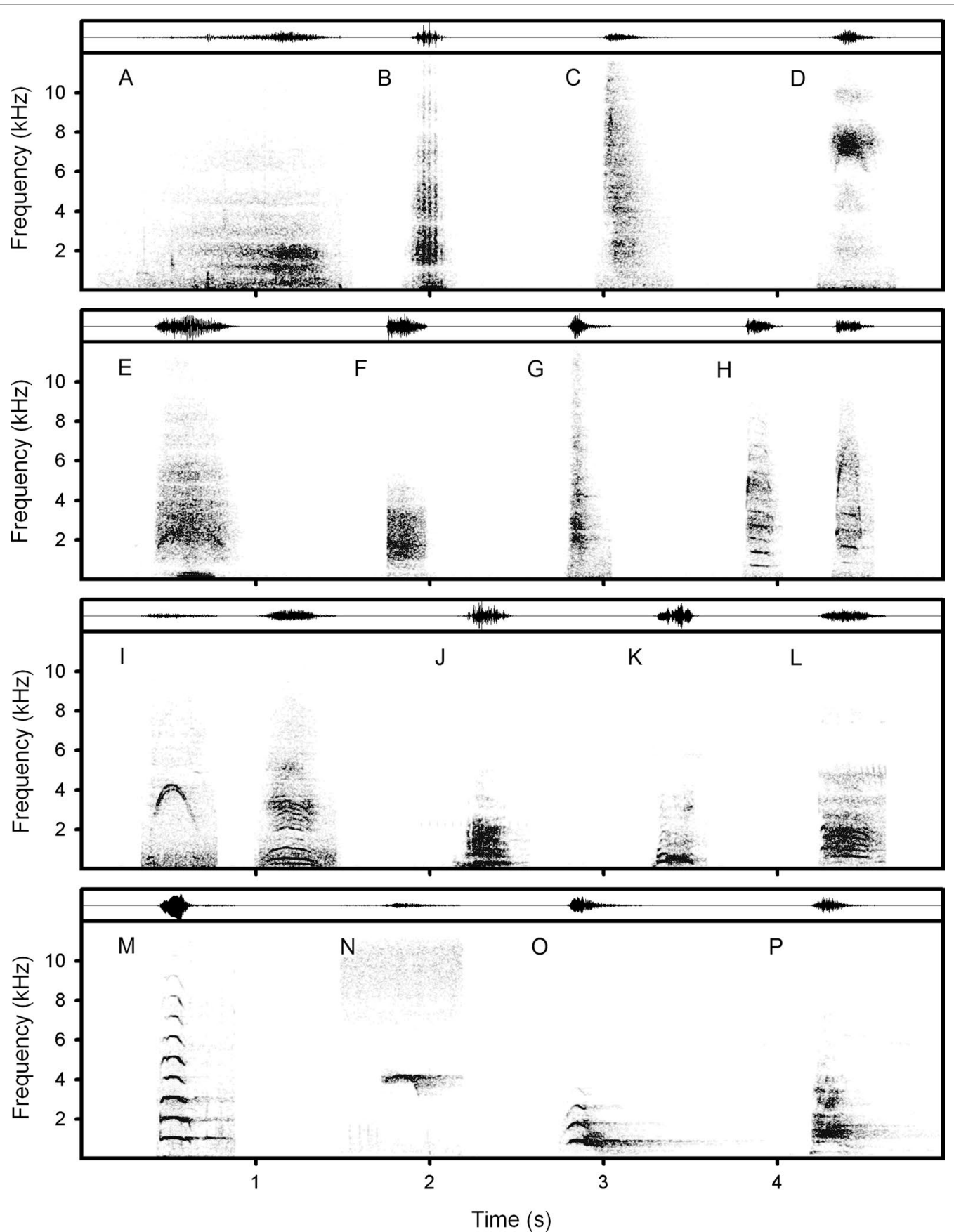

Fig. 2 Vigilance-related calls across Ruminantia. A Hiss and $\mathbf{B}$ snort of a giraffe; $\mathbf{C}$ hiss of a musk deer Moschus moschiferus; $\mathbf{D}$ snort of a female goitred gazelle Gazella subgutturosa; $\mathbf{E}$ snort of a waterbuck Kobus ellipsiprymnus; $\mathbf{F}$ snort of a male impala Aepyceros melampus; $\mathbf{G}$ snort of a male Western tur Capra caucasica cylindricornis; $\mathbf{H}$ two snorts of a male klipspringer Oreotragus oreotragus; I two snorts of a male springbok Antidorcas marsupialis; $\mathbf{J}$ bark of a female greater kudu Tragelaphus strepsiceros; $\mathbf{K}$ bark of a white-tailed gnu Connochaetes gnou; $\mathbf{L}$ bark of a male Indian muntjac Muntiacus vaginalis; $\mathbf{M}$ bark of a female sambar deer Rusa unicolor; $\mathbf{N}$ bark of a female sika deer Cervus nippon; $\mathbf{O}$ bark of a female Siberian red deer Cervus elaphus sibiricus; P bark of a female Bactrian red deer Cervus elaphus bactrianus. The illustrative spectrograms are based on calls recorded from adult wild-living animals (except the Western tur, recorded in captivity) that vocalized at the sudden appearance of a human. During their vocalizations, the callers did not flee but either froze or slowly passed by the human. Spectrograms were created with $24 \mathrm{kHz}$ sampling frequency, Hamming window, Fast Fourier Transform (FFT) 1024 points, frame 50\% and overlap 93.75\%. Original wav-files are available in the electronic supporting information (Additional file 3: Audio S2) 
and measured its acoustic characteristics. The acoustics of this snort (duration $=0.21 \mathrm{~s}$; fpeak $=0.09 \mathrm{kHz}$; q25 $=1.05 \mathrm{kHz}$; q50 = $1.68 \mathrm{kHz}$; $75=3.09 \mathrm{kHz}$; pulse rate $=26.5 \mathrm{~Hz}$ ) were similar to those of the snorts measured in the current study (Table 1).

Giraffe nasal hisses and snorts can be compared by their acoustic structure with vigilance-related nasal hisses and snorts of other ruminants (Fig. 2, Additional file 4: Table S2). Giraffe hisses were the longest calls among ruminants, either nasal or oral (Additional file 4: Table S2). Probably this was the effect of the non-explosive air expulsion during the hisses and/or the effect of large body size (lung volumes) of the giraffe [56]. Similarly non-explosive air expulsion occurs only during the hisses of the much smaller-sized musk deer Moschus moschiferus (Fig. 2). Giraffe snorts had the lowest peak frequency among the snorts of other ruminants (Additional file 4: Table S2). This might also result from the giraffe's large body size [56] and its respectively large vocal tract or larynx $[57,58]$.

Giraffes share the nasal vocal emission with goitred gazelle Gazella subgutturosa [35], impala Aepyceros melampus, Western tur Capra caucasica cylindricornis, klipspringer Oreotragus oreotragus and springbok Antidorcas marsupialis (Additional file 4: Table S2). These nasal calls are probably produced aerodynamically by vortices at the glottis or vocal tract narrowings [59-62], distinctive to oral calls (barks) of, e.g. white-tailed gnu Connochaetes gnou, sambar deer Rusa unicolor and sika deer Cervus nippon (Fig. 2, Additional file 4: Table S2) displaying fundamental frequency produced evidently by the vocal folds $[63,64]$. In the springbok and klipspringer, superimposed fundamental frequency and aerodynamic whistle (Fig. 2, see also [36]) suggest biphonation, described for some cervids $[24,62,65]$. In the giraffe, the fundamental frequency was only reported for the humm vocalization [43], described based on automated recordings in the absence of researchers, so nasal or oral vocal emission and context of this vocalization could not be determined.

\section{Limitations}

This pilot study had a few limitations:

- The study was conducted in one locality in Namibia and one in Kenya, what limits expansion of results for entire local populations of giraffes.

- Context of vocalizing could not be predicted and standardized, as giraffes vocalize very rarely. Two months of stay in Namibia (three researchers, 4-8 h of observations/day) and 1 year of stay in Kenya (one researcher, $8 \mathrm{~h}$ of observations/day) provided only five recording sessions.
- Only 5 of the 9 callers were sexed as vision was complicated by the foliage or twilight during observations in Namibia.

\section{Additional files}

Additional file 1: Audio S1. Hisses and snorts of giraffes emitted toward potential threat in Namibia and Kenya. Hiss of an adult giraffe of unspecified sex from Namibia; hiss of a subadult male giraffe from Kenya; snort of an adult giraffe of unspecified sex from Namibia; snort of an adult female giraffe from Kenya.

Additional file 2: Table S1. Table with acoustic measurements of giraffe hisses and snorts for describing the acoustic features of these two call types.

Additional file 3: Audio S2. Hiss and snort of a giraffe; hiss of a musk deer; snort of a female goitred gazelle; snort of a waterbuck; snort of a male impala; snort of a male Western tur; two snorts of a male klipspringer; two snorts of a male springbok; bark of a female greater kudu; bark of a white-tailed gnu; bark of a male Indian muntjac; bark of a female sambar deer; bark of a female sika deer; bark of a female Siberian red deer; bark of a female Bactrian red deer.

Additional file 4: Table S2. Indicating call type, nasal/oral vocal emission and acoustic characteristics of vigilance-related vocalizations across Ruminantia.

\section{Abbreviations}

fpeak: call maximum amplitude frequency; q25: call lower power quartile; q50: call medium power quartile; q75: call upper power quartile.

\section{Authors' contributions}

EVV and IAV proposed the idea of the study. IAV and EVC secured funding. EVV, EVC, IAV and RF collected data. IAV conducted the bioacoustical and statistical analyses. RF, EV, IAV provided scientific input for the study design and prepared the manuscript draft. All authors reviewed/commented the draft. All authors read and approved the final manuscript.

\section{Author details \\ ${ }^{1}$ Scientific Research Department, Moscow Zoo, Moscow 123242, Russia. 2 Department of Vertebrate Zoology, Faculty of Biology, Lomonosov Moscow State University, Moscow 119234, Russia. ${ }^{3}$ Mara-Meru Cheetah Project, Sarit Centre, P.O. Box 1611, Nairobi 00606, Kenya. ${ }^{4}$ Department of Reproduction Management, Leibniz Institute for Zoo and Wildlife Research, 10315 Berlin, Germany.}

\section{Acknowledgements}

We are grateful to the Okambara Ranch owner Christian Schmitt, for his support and also to Vera Mendes, Ruben Portas, Jörg Melzheimer, Jakob HaiHáb for their invaluable help while we conducted our research at ranch in Namibia. We express gratitude to the Kenya Wildlife Service, Maasai-Mara National Reserve and Mara Conservancy authorities for permission to conduct research in the areas, and to Roman Balaz for providing the equipment for the study in Kenya. We thank the anonymous reviewer for the valuable comments.

\section{Competing interests}

The authors declare that they have no competing interests.

\section{Availability of data and materials}

The dataset supporting the conclusions of this article is included within the article and its additional files.

\section{Consent for publication}

Not applicable.

\section{Ethics approval and consent to participate}

This non-experimental research meets all applicable laws of Namibia and Kenya and was conducted in accordance with the Guidelines for the 
Treatment of Animals in Behavioural Research and Teaching [66]. The nature of the study was purely observational; call collectors did not manipulate the animals for the purpose of this study. No invasive methodologies were applied at any point of the study. The research did not affect the daily routine, behaviour, diet or management of the animals. Disturbance of the animals was kept to a minimum. No animal has suffered somehow due to the data collection. The authors had permissions for data collection of the owner of Okambara Ranch (Namibia) and of the Kenya Wild life Service (Kenya). Giraffes are not endangered in the localities where the data were collected. All methods of data collection were in agreement with the research protocol \# 2011-36 that has been approved by the Committee of Bio-ethics of Lomonosov Moscow State University

\section{Funding}

This study was supported by Russian Science Foundation (http://www.rscf. ru/), Grant Number 14-14-00237, funding to EVV and IAV for work in Namibia and by Roman Wildlife Foundation (http://www.romanwildlifefoundation. com) to EVC, for work in Kenya.

\section{Publisher's Note}

Springer Nature remains neutral with regard to jurisdictional claims in published maps and institutional affiliations.

Received: 2 November 2017 Accepted: 18 December 2017

Published online: 09 January 2018

\section{References}

1. Fuong $\mathrm{H}$, Keeley KN, Bulut $\mathrm{Y}$, Blumstein DT. Heterospecific alarm call eavesdropping in nonvocal, white-bellied copper-striped skinks, Emoia cyanura. Anim Behav. 2014;95:129-35

2. Seyfarth RM, Cheney DL, Marler P. Vervet monkey alarm calls: semantic communication in a free-ranging primate. Anim Behav. 1980;28:1070-94.

3. Zuberbühler K. Survivor signals: the biology and psychology of animal alarm calling. Adv Study Behav. 2009;40:277-322.

4. Townsend SW, Charlton BD, Manser MB. Acoustic cues to identity and predator context in meerkat barks. Anim Behav. 2014;94:143-9.

5. Price T, Wadewitz P, Cheney D, Seyfarth R, Hammerschmidt K, Fischer J. Vervets revisited: a quantitative analysis of alarm call structure and context specificity. Sci Rep. 2015;5:13220.

6. Briefer EF. Vocal expression of emotions in mammals: mechanisms of production and evidence. J Zool. 2012;288:1-20.

7. Rauber R, Manser MB. Discrete call types referring to predation risk enhance the efficiency of the meerkat sentinel system. Sci Rep. 2017;7:44436.

8. Fichtel C, Kappeler PM. Anti-predator behavior of group-living Malagasy primates: mixed evidence for a referential alarm call system. Behav Ecol Sociobiol. 2002;51:262-75.

9. Shelley EL, Blumstein DT. The evolution of vocal alarm communication in rodents. Behav Ecol. 2005;16:169-77.

10. Townsend SW, Rasmussen M, Clutton-BrockT, Manser MB. Flexible alarm calling in meerkats: the role of the social environment and predation urgency. Behav Ecol. 2012;23:1360-4.

11. Sherman PW. Nepotism and the evolution of alarm calls. Science. 1977;197:1246-53.

12. Pipia A, Ciuti S, Grignolio S, Luchetti S, Madau R, Apollonio M. Effect of predation risk on grouping pattern and whistling behaviour in a wild mouflon Ovis aries population. Acta Theriol. 2009:54:77-86.

13. Kitchen D, Bergman T, Cheney D, Nicholson J. Comparing responses of four ungulate species to playbacks of baboon alarm calls. Anim Cogn. 2010;13:861-70.

14. Magrath RD, HaffTM, Fallow PM, Radford AN. Eavesdropping on heterospecific alarm calls: from mechanisms to consequences. Biol Rev Camb Philos Soc. 2015;90:560-86.

15. Matrosova VA, Blumstein DT, Volodin IA, Volodina EV. The potential to encode sex, age, and individual identity in the alarm calls of three species of Marmotinae. Naturwissenschaften. 2011;98:181-92.
16. García-Navas V, Blumstein DT. The effect of body size and habitat on the evolution of alarm vocalizations in rodents. Biol J Linn Soc. 2016:118:745-51.

17. Riede T, Zuberbühler K. The relationship between acoustic structure and semantic information in Diana monkey alarm vocalization. J Acoust Soc Am. 2003;114:1132-42.

18. Townsend SW, Manser MB. The function of non-linear phenomena in meerkat alarm calls. Biol Lett. 2011;7:47-9.

19. Kern JM, Radford AN. Call of duty? Variation in use of the watchman's song by sentinel dwarf mongooses, Helogale parvula. Anim Behav. 2013;85:967-75.

20. Déaux EC, Allen AP, Clarke JA, Charrier I. Concatenation of 'alert' and 'identity' segments in dingoes' alarm calls. Sci Rep. 2016;6:30556.

21. Struhsaker TT. Behavior of the elk (Cervus canadensis) during the rut. $Z$ Tierpsychol. 1968;24:80-114.

22. Bowyer TR, Kitchen DW. Sex and age-class differences in vocalization of Roosevelt elk during rut. Am Midl Nat. 1987;118:225-35.

23. Long AM, Moore NP, Hayden TJ. Vocalizations in red deer (Cervus elaphus), sika deer (Cervus nippon), and red $\times$ sika hybrids. J Zool. 1998;224:123-34.

24. Volodin IA, Volodina EV, Frey R, Maymanakova IL. Vocal activity and acoustic structure of the rutting calls of Siberian wapiti (Cervus elaphus sibiricus) and their imitation with a hunting luring instrument. Russ J Theriol. 2013;12:99-106.

25. Minami M, Kawamichi T. Vocal repertoires and classification of the sika deer Cervus nippon. J Mammal Soc Japan. 1992;17:71-94.

26. Richardson LW, Jacobson HA, Muncy RJ, Perkins CJ. Acoustics of whitetailed deer (Odocoileus virginianus). J Mammal. 1983:64:245-52.

27. Atkeson TD, Marchinton RL, Miller KV. Vocalizations of white-tailed deer Am Midl Nat. 1988:120:194-200.

28. Volodin IA, Volodina EV, Frey R, Gogoleva SS, Palko IV, Rozhnov WV. Acoustic structure of alarm calls in Indian sambar (Rusa unicolor) and Indian muntjac (Muntiacus vaginalis) in South Vietnam. Dokl Biol Sci. 2017:474:110-3.

29. Oli MK, Jacobson HA. Vocalizations of barking deer (Muntiacus muntjak) in Nepal. Mammalia. 1995;59:179-86.

30. Yahner R. Barking in a primitive ungulate, Muntiacus reevesi: function and adaptiveness. Am Nat. 1980;116:157-77.

31. Sokolov VE, Prikhod'ko VI, Rutovskaya MV. Vocalizations in the musk deer (Moschus moschiferus). Zool Zh. 1984;63:263-71.

32. Sokolov VE, Gromov VS, Rutovskaya MV. Sound signalization in the European (Capreolus capreolus) and Siberian (C. pygargus) roe deer. Zool Zh. 1987;66:430-43.

33. Reby D, Cargnelutti B, Joachim J, Aulagnier S. Spectral acoustic structure of barking in roe deer (Capreolus capreolus). Sex-, age- and individual related variations. C R Acad Sci Paris. 1999;322:271-9.

34. Bro-Jørgensen J, Pangle WM. Male topi antelopes alarm snort deceptively to retain females for mating. Am Nat. 2010;176:33-9.

35. Efremova KO, Volodin IA, Volodina EV, Frey R, Lapshina EN, Soldatova NV. Developmental changes of nasal and oral calls in the goitred gazelle Gazella subgutturosa, a nonhuman mammal with a sexually dimorphic and descended larynx. Naturwissenschaften. 2011:98:919-31.

36. Tilson RL, Norton PM. Alarm duetting and pursuit deterrence in an African antelope. Am Nat. 1981;118:455-62.

37. Sharma K, Rahmani AR, Chundawat RS. Natural history observations of the four-horned antelope Tetracerus quadricornis. J Bombay Nat Hist Soc. 2009;106:72-82.

38. Langman VA. Cow-calf relationships in giraffe (Giraffa camelopardalis giraffa). ZTierpsychol. 1977;43:264-86

39. Hurgitsch B. Akustische Kommunikation und Mutter-Kalb-Beziehung bei Giraffen (Giraffa camelopardalis) in zoologischer Haltung (in German) (Master's thesis). Vienna: University of Vienna; 2011. p. 56.

40. Dagg Al. Individual behaviours. In: Giraffe: biology, behaviour and conservation. Cambridge: Cambridge University Press; 2014. p. 84

41. von Muggenthaler E, Harris K, Baes C, Fulk R. Infrasound and low frequency vocalizations from the giraffe; Helmholtz resonance in biology. In: Proceedings of the riverbanks research consortium on biology and behaviour. 1999.

42. von Muggenthaler E. Giraffe Helmholtz resonance. Proc Meet Acoust. 2013;19:010012. 
43. Baotic A, Sicks F, Stoeger AS. Nocturnal "humming" vocalizations: adding a piece to the puzzle of giraffe vocal communication. BMC Res Notes. 2015;8:425.

44. Shorrocks B. The giraffe: biology, ecology, evolution and behaviour. Hoboken: Wiley-Blackwell; 2016. p. 232.

45. Dagg Al, Foster JB. The giraffe: its biology, behaviour and ecology. New York: Van Nostrand Reinhold Co.i 1976. p. 210.

46. Dagg Al. Giraffa camelopardalis. Mammal Species. 1971;5:1-8.

47. Seeber PA, Ciofolo I, Ganswindt A. Behavioural inventory of the giraffe (Giraffa camelopardalis). BMC Res Notes. 2012;5:650.

48. Brown DM, Brenneman RA, Koepfli K-P, Pollinger JP, Milá B, Georgiadis $\mathrm{NJ}$, et al. Extensive population genetic structure in the giraffe. BMC Biol. 2007;5:57.

49. Hayward MW, Hofmeyr M, O'Brien J, Kerley GIH. Prey preferences of the cheetah (Acinonyx jubatus) (Felidae: Carnivora): morphological limitations or the need to capture rapidly consumable prey before kleptoparasites arrive? J Zool. 2006;270:615-27.

50. Hunter L, Hamman D. Cheetah. Cape Town: Struik Nature; 2007. p. 144

51. Ishengoma E, Agaba M, Cavener DR. Evolutionary analysis of vision genes identifies potential drivers of visual differences between giraffe and okapi. PeerJ. 2017;5(e3145):2017.

52. Pereira L. Olfactory discrimination in a captive reticulated giraffe: (Giraffo camelopardalis reticulata). Wheaton College Digital Repository, Master thesis. 2012; p. 106. http://hdl.handle.net/11040/23773.

53. Wilson DR, Hare JF. Ground squirrel uses ultrasonic alarms. Nature. 2004:430:523.

54. Wilson DR, Hare JF. The adaptive utility of Richardson's ground squirrel (Spermophilus richardsonii) short-range ultrasonic alarm signals. Can J Zool. 2006:84:1322-30.
55. Cunningham S, Magrath RD. Functionally referential alarm calls in noisy miners communicate about predator behaviour. Anim Beh. 2017:129:171-9.

56. Gillooly JF, Ophir AG. The energetic basis of acoustic communication. Proc R Soc B. 2010;277:1325-31.

57. Harrison DF. Biomechanics of the giraffe larynx and trachea. Acta Otorhinolaryngol. 1980;89:258-64.

58. Erdoğan S, Pérez W. Anatomical characteristics of the larynx in giraffe (Giraffa camelopardalis). J Morphol Sci. 2013;30:266-71.

59. Chanaud RC. Aerodynamic whistles. Sci Am. 1970;222:40-6.

60. Frey $R$, Riede T. The anatomy of vocal divergence in North American elk and European red deer. J Morphol. 2013;274:307-19.

61. Frey R, Volodin IA, Fritsch G, Volodina EV. Potential sources of high frequency and biphonic vocalization in the dhole (Cuon alpinus). PLOS ONE. 2016;11(1):e0146330

62. Reby D, Wyman MT, Frey R, Passilongo D, Gilbert J, Locatelli Y, Charlton $\mathrm{BD}$. Evidence of biphonation and source-filter interactions in the bugles of male North American wapiti (Cervus canadensis). J Exp Biol. 2016;219:1224-36.

63. Titze IR. Principles of voice production. NJ: Prentice Hall, Englewood Cliffs; 1994. p. 354.

64. Wilden I, Herzel H, Peters G, Tembrock G. Subharmonics, biphonation, and deterministic chaos in mammal vocalization. Bioacoustics. 1998;9:171-96.

65. Golosova OS, Volodin IA, Isaeva IL, Volodina EV. Effects of free-ranging, semi-captive and captive management on the acoustics of male rutting calls in Siberian wapiti Cervus elaphus sibiricus. Mammal Res. 2017:62:387-96.

66. Guidelines for the treatment of animals in behavioural research and teaching. Anim Behav. 2006; 71: 245-253.

\section{Submit your next manuscript to BioMed Central and we will help you at every step:}

- We accept pre-submission inquiries

- Our selector tool helps you to find the most relevant journal

- We provide round the clock customer support

- Convenient online submission

- Thorough peer review

- Inclusion in PubMed and all major indexing services

- Maximum visibility for your research

Submit your manuscript at www.biomedcentral.com/submit
O Biomed Central 